\title{
04.1
}

\section{Энергия поверхностного разряда в электродной системе параллельных полос}

\author{
(c) А.В. Лазукин, А.М. Никитин, Г.А. Романов \\ Национальный исследовательский университет „Московский энергетический институт“, Москва, Россия \\ E-mail: lazukin_av@mail.ru
}

Поступило в Редакцию 7 апреля 2021 г.

В окончательной редакции 7 июня 2021 г.

Принято к публикации 21 июня 2021 г.

\begin{abstract}
Приведены результаты экспериментального исследования зависимости энергии поверхностного барьерного разряда от значения питающего синусоидального напряжения частотой 4 и $20 \mathrm{kHz}$ в электродной системе параллельных полос с расстоянием между ними 5 и $30 \mathrm{~mm}$. При расстоянии $5 \mathrm{~mm}$ между полосами в электродной системе существует самоограниченный поверхностный разряд. Самоограничение разряда приводит к тому, что с повышением приложенного напряжения энергия, вложенная в электродную систему, возрастает медленнее, чем в случае, когда разряд существует без ограничений. Энергия самоограниченного разряда ниже на $15-40 \%$ энергии разряда, существующего без ограничения.
\end{abstract}

Ключевые слова: поверхностный разряд, энергия, вольт-кулонная характеристика, самоограниченный разряд.

DOI: 10.21883/PJTF.2021.19.51505.18812

Поверхностный разряд - это разновидность газового разряда атмосферного давления, существующего вблизи поверхности диэлектрика около электрода с малым радиусом кривизны. Диэлектрик разделяет электрод с малым радиусом кривизны и второй электрод системы, между которыми приложено переменное напряжение. Поверхностный разряд в настоящее время используется как основа ряда электротехнологий с применением низкотемпературной плазмы, что обусловлено простотой и надежностью электродных систем и источников питания, отсутствием вакуумной части или расхода инертных газов. Наиболее полный список технологических решений с применением поверхностного разряда и других барьерных разрядов можно найти в обзоре [1] и вводных частях работ $[2,3]$.

Для решения технологической задачи (например, при обработке поверхности полимера или дерева, слоя семян и т.п.) возникает необходимость масштабировать электродную систему поверхностного разряда. В этом случае используют системы параллельных полосовых электродов или электроды-сетки (сравнение подобных систем по ряду параметров выполнено в [2]). Соседние полосовые электроды или границы ячейки (в случае сетки) находятся под одним потенциалом. При достижении этого потенциала выше некоторого начального значения с кромок электродов стартует группа микроразрядов (это проиллюстрировано методами высокоскоростной фотографии, например, в работе [3]). Однако если соседние электроды находятся достаточно близко, встречные группы микроразрядов препятствуют развитию друг друга, и поверхностный разряд становится самоограниченным. Дистанция „самоограничения“ определяется конфигурацией электродов и параметрами питающего напряжения [4]. Возникновение самоограничения должно отразиться на энергии разряда.

Цель настоящей работы заключается в выявлении зависимости энергии поверхностного разряда от напряжения и частоты в электродных системах с самоограниченным разрядом.

Энергия поверхностного разряда, вложенная в электродную систему за период питающего напряжения, измерялась методом вольт-кулонных характеристик (ВКХ). Схема измерения и вид электродной системы приведены на рис. 1, $a$. Вид ВКХ для $4 \mathrm{kHz}$ при расстоянии $30 \mathrm{~mm}$ между полосами приведен на рис. 1, $b$.

Электродная система выполнена на диэлектрическом барьере из корундовой керамики ВК-96 толщиной $\Delta=1 \mathrm{~mm}$ в виде двух параллельных полос из алюминиевой фольги (толщина $20 \mu \mathrm{m}$ ). Выполнены две системы с расстоянием между полосами 5 и $30 \mathrm{~mm}$. Противоположную сторону диэлектрического барьера занимает заземленный обратный электрод, в котором выделена измерительная секция шириной $10 \mathrm{~mm}$. Измерительная секция заземляется через конденсатор $C 1$. Параллельно плечу, образованному геометрической емкостью электродной системы и емкостью $C 1$, подключается „компенсирующее“ плечо, состоящее из вакуумного конденсатора переменной емкости $C_{v}$ и емкости $C 2$ (емкости $C 1$ и $C 2$ одинаковы по номиналу: $4 \mathrm{nF}$ ). К средним точкам образованного моста подключается высоковольтный дифференциальный пробник DP (DP-150, Pintek). Таким образом измеряется энергия разряда, существующего только над измерительной секцией, без вклада энергии, вводимой в геометрическую емкость электродной системы. Напряжение контролируется высоковольтным смешанным делителем P6015A (Tektronix). Синусоидальное напряжение частотой 4 или $20 \mathrm{kHz}$ подается 


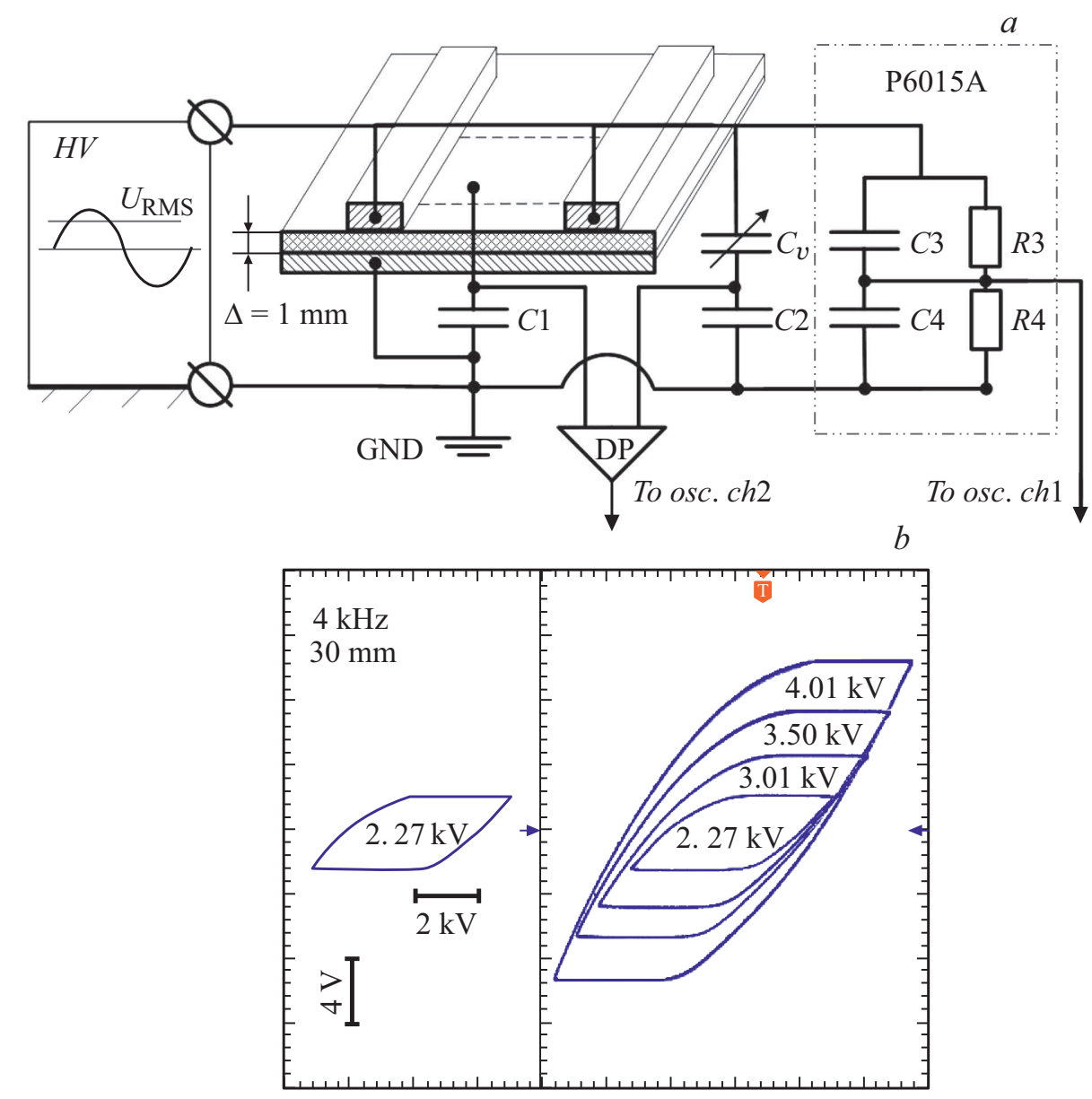

Рис. 1. $a-$ электродная конфигурация и схема измерений; $b-$ вид ВКХ при частоте $4 \mathrm{kHz}$ для разных величин действующего значения напряжения.

от высоковольтного источника $H V$. Источник выполнен в виде полумостового транзисторного инвертора с выходным каскадом на высоковольтном трансформаторе и дросселе. Индуктивность выходного каскада подбирается таким образом, чтобы обеспечить условия, близкие к резонансной работе контура на разрядную нагрузку.

На рис. 2 приведены результаты измерения энергии для 4 и $20 \mathrm{kHz}$ при повышении напряжения от 1.5 до $4 \mathrm{kV}$ (действующее значение). Начальное напряжение составляет $1.4 \mathrm{kV}$. Энергия растет с повышением приложенного напряжения по характерной для разрядов атмосферного давления степенной зависимости, что согласуется с литературными данными [2,5]. Энергия разряда также зависит от частоты питающего напряжения, что противоречит данным, приведенным в работе [2]. По данным [2], где использовалась система с расстоянием $3 \mathrm{~mm}$ между полосами из медной фольги толщиной $35 \mu \mathrm{m}$ при питании синусоидальным напряжением 4.7 и $10 \mathrm{kHz}$, энергия, вложенная в разряд за период питающего напряжения, практически не зависит от частоты. Вероятно, при расстоянии между параллельными полосами в $3 \mathrm{~mm}$ зоны горения встречных разрядов смыкаются практически на напряжениях зажигания.
Длины разрядных зон в подобных системах приведены в работе [4], где обстоятельства самоограничения поверхностного разряда оценивались по высокоскоростным фотографиям. В системе параллельных полос с расстоянием $5 \mathrm{~mm}$ между ними встречные микроразряды начинают влиять друг на друга при напряжении $2.5 \mathrm{kV}$. Частота питающего напряжения также влияет на обстоятельства самоограничения. На $20 \mathrm{kHz}$ разрядные зоны смыкаются при $2.5 \mathrm{kV}$. На $4 \mathrm{kHz}$ полное смыкание разрядных зон наблюдается при напряжениях около $3 \mathrm{kV}$. Это обстоятельство должно отразиться на соотношении энергий таких разрядов в зависимости от частоты.

Отношение энергий разрядов при 5 и $30 \mathrm{~mm}$ между полосами в зависимости от напряжения приведено на рис. 3. При переходе к электродной системе поверхностного разряда с расстоянием $5 \mathrm{~mm}$ между параллельными электродами энергия разряда оказывается меньше, чем в случае $30 \mathrm{~mm}$ между электродами. С ростом напряжения отношение энергий снижается на 15-40\%. Характер снижения зависит от частоты. Для $20 \mathrm{kHz}$ отношение энергий снижается до $0.8-0.9$ и держится в этом диапазоне при повышении напряжения от 2 до $3 \mathrm{kV}$. $\mathrm{C}$ дальнейшим повышением напряжения от 3 до $4 \mathrm{kV}$ от- 


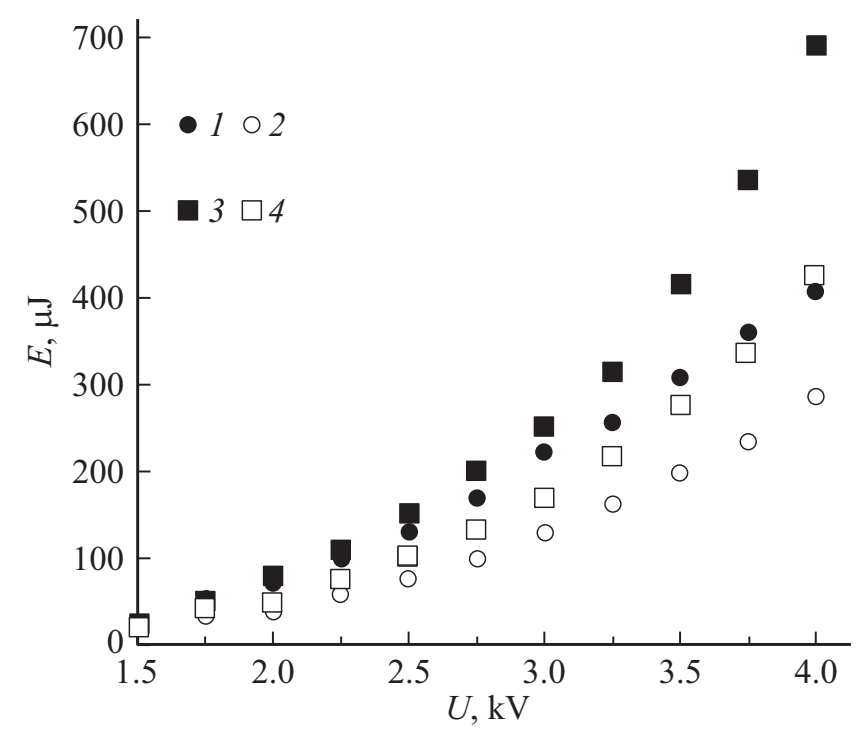

Рис. 2. Энергия поверхностного разряда в зависимости от напряжения. Темные символы - для $20 \mathrm{kHz}$, светлые - для $4 \mathrm{kHz} .1,2-5 \mathrm{~mm}$ между полосами; $3,4-30 \mathrm{~mm}$ между полосами.

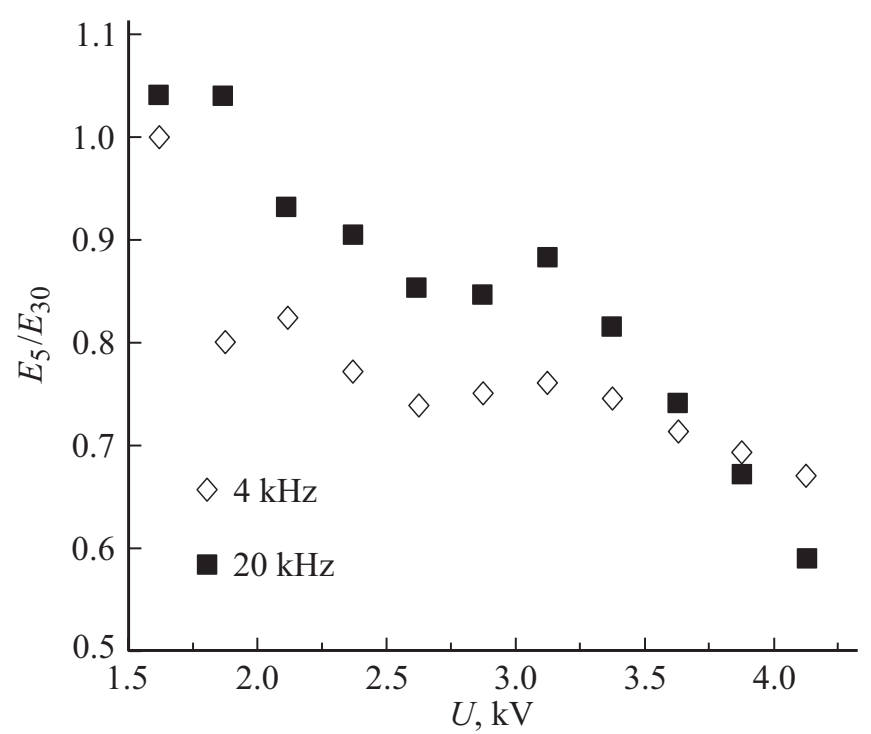

Рис. 3. Отношение энергии разряда при расстоянии между полосами $5 \mathrm{~mm}\left(E_{5}\right)$ к энергии разряда при расстоянии между полосами $30 \mathrm{~mm}\left(E_{30}\right)$ на частотах 4 и $20 \mathrm{kHz}$.

ношение энергий снижается линейно до 0.6. Для $4 \mathrm{kHz}$, кроме начального этапа снижения отношения энергий от 1 до 0.8 при повышении напряжения от 1.5 до $1.75 \mathrm{kV}$, отношение энергий снижается практически линейно с ростом приложенного напряжения от 0.8 до 0.67 .

На основе полученных зависимостей можно сделать следующие выводы. Энергия, вложенная в системы с самоограниченным разрядом, не выходит на насыщение в зависимости от напряжения, однако растет не так быстро, как энергия разряда, развивающегося свободно. Вероятно, увеличивается число отдельных микроразря- дов вдоль кромки, но не увеличивается переносимый в индивидуальном импульсе заряд. На $4 \mathrm{kHz}$ подобная реорганизация разрядных структур проходит лучшим образом, чем на $20 \mathrm{kHz}$. Следовательно, для систем с самоограниченным разрядом более предпочтительны невысокие частоты питающего напряжения.

C точки зрения технологического применения поверхностного разряда важна не только энергия разряда, но и другие электрофизические характеристики, например интенсивность образования объемного заряда или плотность УФ-потока. Подробное исследование связи энергии самоограниченного поверхностного разряда с ключевыми технологическими характеристиками электродных систем предполагается провести в дальнейшем.

\section{Финансирование работы}

Работа выполнена при финансовой поддержке Российского научного фонда (проект № 18-76-10019).

\section{Конфликт интересов}

Авторы заявляют, что у них нет конфликта интересов.

\section{Список литературы}

[1] R. Brandenburg, Plasma Sources Sci. Technol., 26, 053001 (2017). DOI: 10.1088/1361-6595/aa6426

[2] S. Pekárek, J. Mikeš, Eur. Phys. J. D, 74, 59 (2020). DOI: $10.1140 /$ epjd/e2020-100332-3

[3] I. Selivonin, I. Moralev, Plasma Sources Sci. Technol., 30 (3), 035005 (2021). DOI: 10.1088/1361-6595/abe0a1

[4] А.В. Лазукин, И.В. Селивонин, М.Э. Пинчук, И.А. Моралев, С.А. Кривов, Изв. вузов. Физика, 61 (9-2), 152 (2018). https://elibrary.ru/item.asp?id $=36614175$

[5] J. Kriegseis, B. Möller, S. Grundmann, C. Tropea, J. Electrostat., 69, 302 (2011). DOI: 10.1016/j.elstat.2011.04.007 